\title{
TRANSVERSE INSTABILITY OF A RECTANGULAR BUNCH *
}

\author{
V. Balbekov, FNAL, Batavia, IL 60510, USA
}

\section{Abstract}

Transverse instability of a rectangular bunch is investigated. Known theory of bunched beam instability is modified to take into account $100 \%$ spread of synchrotron frequency. Series of equations adequately describing the instability is derived and solved analytically and numerically. The theory is applied to the Fermilab Recycler Ring.

\section{INTRODUCTION}

Transverse beam instability - probably resistive wall type - is observed sometimes in the Fermilab Recycler Ring $[1,2]$. A damper is designed now to avoid problems at planned increase of the Recycler intensity. It should be taken into account that, typically, the Recycler beam is bunched longitudinally by a barrier-bucket rf waveform. Therefore the bunch has almost rectangular shape, synchrotron frequency is rather low and has 100\% spread. One would expect that instability of such beam is a cross between a coasting beam and "normal" bunched beam. Theoretical investigation of this problem and development of requirements to the damper is the main subject of this paper. In doing so, we neglect moderate effects of penetration of particles into the barriers, and nonlinearity of betatron oscillations.

\section{BASIC EQUATIONS}

Let us consider transverse dipole moment of a beam $D(\theta) \exp (-i \omega t)$ where $\theta$ is azimuth in a rest frame. Its Fourier harmonics satisfy the set of equations (see e.g. [3]):

$$
\begin{aligned}
D_{k} & =\frac{i r_{0} \omega_{0} N}{2 \pi \gamma Q_{0} Z_{0}} \sum_{k^{\prime}} C_{k, k^{\prime}} D_{k^{\prime}} Z_{k^{\prime}}^{\perp} \\
C_{k, k^{\prime}} & =\sum_{m} \int_{0}^{\infty} \frac{I_{m, k}(\epsilon) I_{m, k^{\prime}}^{*}(\epsilon) F(\epsilon) d \epsilon}{\omega+\omega_{0} Q_{0}-m \Omega}
\end{aligned}
$$

where $N$ is the beam intensity, $\omega_{0}$ and $Q_{0}$ are average angular velocity and betatron frequency, $\epsilon$ and $\Omega(\epsilon)-$ action and frequency of synchrotron oscillations, $F(\epsilon)-$ normalized distribution function, $Z_{k}^{\perp}-$ transverse beam coupling impedance [4], $r_{0}=e^{2} / m c^{2} \simeq 1.535 \times$ $10^{-16} \mathrm{~cm}, Z_{0}=4 \pi / c \simeq 376.7 \mathrm{Ohm}$. General formula for form-factors $I_{m, k}$ is

$$
I_{m, k}(\epsilon)=\frac{1}{2 \pi} \int_{-\pi}^{\pi} e^{i m \phi-i \lambda \theta(\epsilon, \phi)} d \phi
$$

\footnotetext{
* Work supported by the U. S. Department of Energy under contract DE-AC02-76CH03000.
}

where $\lambda=k-Q_{0}+\xi / \eta, \xi$ and $\eta$ are chromaticity and slippage factor, and azimuth $\theta$ is presented as a function of action and phase of synchrotron oscillations.

In a rectangular potential well of the length $\theta_{0}, \quad \theta=$ $2 B|\phi|, \epsilon=4 \pi B\left|p-p_{0}\right|$, and

$$
\Omega=\frac{\omega_{0}\left|\eta\left(p-p_{0}\right)\right|}{2 p_{0} B}=\frac{\omega_{0} \epsilon|\eta|}{8 \pi p_{0} B^{2}}
$$

where $p$ and $p_{0}$ are the particle momentum and central one, and $B=\theta_{0} / 2 \pi$ is a bunch factor. Then formula (3) gives:

$$
I_{m, k}=\frac{\psi_{m, k}+\pi m / 2}{\psi_{m, k}+\pi m} \frac{\sin \psi_{m, k}}{\psi_{m, k}} e^{i \psi_{m, k}}
$$

where $\psi_{m, k}=\pi(B \lambda-m / 2)$. The sum over $m$ in Eq. (2) is calculated analytically in this case providing general formula for the matrix $C_{k, k^{\prime}}$. However, it is very cumbersome; therefore we will restrict our consideration to the required specific cases.

\section{LOW SYNCHROTRON FREQUENCY}

As the first approximation, consider the case when both $\eta$ and $\Omega \rightarrow 0$. It is reasonable because typical synchrotron frequency in the Recycler is several $\mathrm{Hz}$ whereas the space charge tune shift can reach several hundred $\mathrm{Hz}$. The result is: ${ }^{1}$

$$
C_{k, k^{\prime}}=N_{k-k^{\prime}} \int_{-\infty}^{\infty} \frac{\mathcal{F}(p) d p}{\omega+\omega_{0} Q(p)}
$$

where $\mathcal{F}(p)$ is normalized distribution function on momentum, $Q(p)=Q_{0}+\xi\left(p-p_{0}\right) / p_{0}$ is the momentumdepending tune, and

$$
N_{l}=\frac{\sin (\pi l B)}{\pi l B} e^{i \pi l B}
$$

is Fourier harmonic of normalized beam density which is $1 / B$ in the potential well. Therefore dispersion equation of the instability can be written in the form:

$$
1=\frac{i r_{0} \omega_{0} N Z^{(e f)}}{2 \pi \gamma Q_{0} Z_{0}} \int_{-\infty}^{\infty} \frac{\mathcal{F}(p) d p}{\omega+\omega_{0} Q(p)}
$$

what is quite similar to the equation for a coasting beam at $\eta=0$. The only distinction is a replacement of the beam coupling impedance $Z_{k}^{\perp}$ by an effective impedance $Z^{(e f)}$ which is defined here as any eigenvalue of the problem:

$$
Z^{(e f)} D_{k}=\sum_{k^{\prime}} N_{k-k^{\prime}} Z_{k^{\prime}}^{\perp} D_{k^{\prime}}
$$

At $B=1$, the distinction entirely disappears because $Z^{(e f)}=Z_{k}^{\perp}$ at $N_{k-k^{\prime}}=\delta_{k, k^{\prime}}$. Some results of numerical solution at $B \neq 1$ are represented below.

\footnotetext{
${ }^{1}$ Note that it would be wrong simply to take $\Omega=0$ in Eq. (2) because parameter $\lambda \rightarrow \infty$ at the same time.
} 


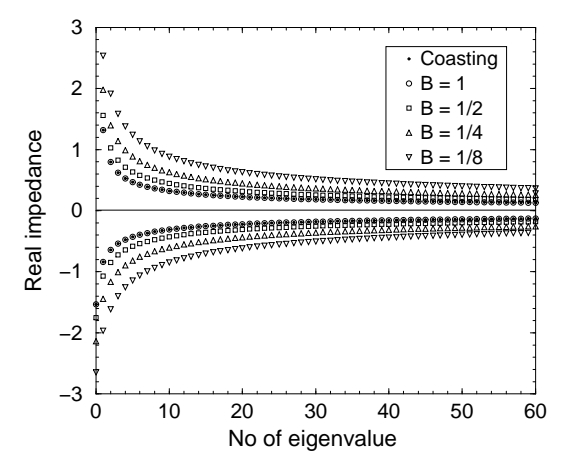

Figure 1: Real part of the effective resistive wall impedance

\section{Resistive wall impedance}

First example considered is resistive wall impedance:

$$
Z_{k}^{\perp}=Z^{(r w)} \frac{\operatorname{sgn}(\kappa)-i}{\sqrt{|\kappa|}}
$$

where $\kappa=k+\omega / \omega_{0} \simeq k-Q_{0}$ is normalized frequency in the laboratory frame [4]. Real parts of the eigenvalues are presented in Fig. 1 at $Z^{(r w)}=1, Q_{0}=0.425$, and different $B$. They are arranged in order of decreasing absolute value, which in practice is the same as increasing number of central harmonic of the spectrum. At $B=1$, the numerical solution coincides with analytical solution of a coasting beam. Note that approximate dependence of varied eigenvalues on bunch factor is between $B^{-0.3}-B^{-0.5}$.

\section{Exponential wake}

Another important case is the impedance

$$
Z_{k}^{\perp}=\frac{Z^{(e x)}}{1-i \alpha \kappa}
$$

corresponding an exponentially decreasing wake field. It turns out that in this case $Z_{k}^{(e f)}=Z_{k}^{\perp}$ regardless the bunch factor, though the eigenfunctions essentially depend on $B$ (the result is obtained both analytically and numerically).

\section{Resistive wall and damper}

Combining Eq. (10) and (11) with real negative $Z^{(e x)}$, one can get a model of an accelerator with a resistive wall and simplest wide-band damper:

$$
Z_{k}^{\perp}=Z^{(r w)}\left(\frac{\operatorname{sgn}(\kappa)-i}{\sqrt{|\kappa|}}-\frac{g}{1-i \alpha \kappa}\right)
$$

Solution at $Q_{0}=0.425, Z^{(r w)}=1, \quad \alpha=0.04, g=1.32^{2}$ is illustrated by Fig. 2 where the eigenvalues multiplied on $B$ are placed in complex plane. It is seen that their absolute value increases at the bunching not faster than $1 / B$. It is seen also that $\operatorname{Re} Z^{(e f)}>0$ for higher modes which means that the beam is potentially unstable on high frequency even with such a damper.

\footnotetext{
${ }^{2}$ At this $g$ and $Q_{0}$, the damper exactly suppresses real part of the most dangerous resistive wall harmonic $k=1$.
}

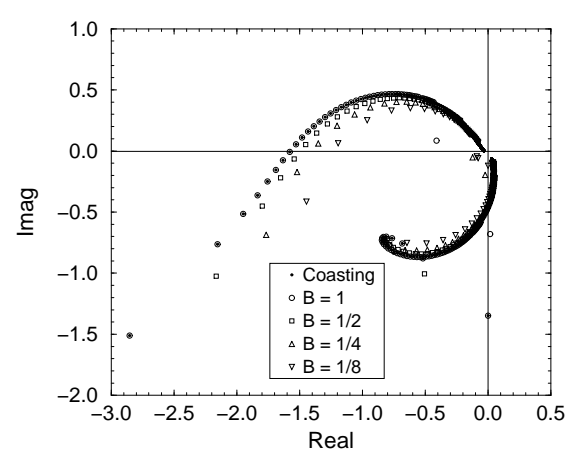

Figure 2: An accelerator with resistive walls and minimal damper: effective impedance multiplied on bunch factor.

\section{UNIFORM BEAM}

The mentioned instability can be suppressed by Landau damping due to spread of incoherent frequency. According to Eq. (8), only chromaticity gives a contribution to the spread which would restrict scope of the method. However, it is a premature conclusion because slippage factor was neglected in those considerations. Doubtless, the factor is especially important at the analysis of higher modes determining requirements to the damper.

In this part we can restrict ourself by the case $B=1$ what means a uniform (not a coasting!) beam. Indeed, it follows from previous that, at adiabatic compression of the beam, the effective impedance increases not faster than momentum and frequency spread, i.e. $\propto 1 / B$. That is true if the space charge impedance $Z_{k}^{\perp}=i Z^{(s c)}$ is taken into account also, adding $i Z^{(s c)} / B$ to any eigenvalue of Eq. (9). Therefore the beam is most unstable at $B=1$ which case forms the requirements to the damper. Then the matrix $C_{k, k^{\prime}}$ is:

$$
\begin{aligned}
& C_{k, k^{\prime}}=\delta_{k, k^{\prime}} \int_{-\infty}^{\infty} \frac{\mathcal{F}(p) d p}{\omega+\omega_{\text {rev }}(p) Q(p)+k \omega_{0} \eta\left(p-p_{0}\right) / p_{0}} \\
& +\int_{-\infty}^{\infty} \frac{\left[\nu+2\left(\lambda+\lambda^{\prime}\right) \Omega\right][\cos (2 \pi \lambda)-\cos (\pi \nu / \Omega)]}{\pi\left[\lambda+\lambda^{\prime}\right][\nu+2 \lambda \Omega]\left[\nu+2 \lambda^{\prime} \Omega\right] \sin (\pi \nu / \Omega)} \mathcal{F}(p) d p
\end{aligned}
$$

First part of the formula coincides with similar expression for a coasting beam and corresponds to an approximation of separated harmonics in Eq. (1). Second part due to the reflection of the particles from the walls is small because the integrand is fast oscillating function at low synchrotron frequency. We can consider it as a small addition to determine limits of applicability of the coasting beam approximation. Then the dispersion equation is:

$$
\frac{i r_{0} \omega_{0} N Z_{k}^{\perp} C_{k, k}}{2 \pi \gamma Q_{0} Z_{0}}=1
$$

Further we will consider Gaussian distribution on momentum with dispersion $\sigma_{p}$, and rewrite Eq. (14) in the form:

$$
\frac{2 \pi i \gamma|\eta \lambda| Q_{0} \sigma_{p} Z_{0}}{r_{0} N p_{0} Z_{k}^{\perp}}=\frac{1}{\sqrt{2 \pi}} \int_{-\infty}^{\infty} \frac{e^{-x^{2} / 2}}{x-x_{\omega}} \times
$$




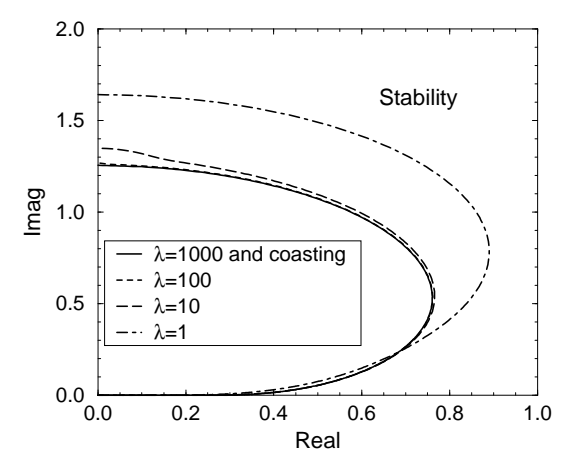

Figure 3: Threshold diagram of a coasting beam and bunched beam at $B=1$.

$$
\left(1+\frac{\left[2 x-x_{\omega}\right]\left[\cos (2 \pi \lambda)-\cos \left(2 \pi \lambda x_{\omega} / x\right)\right]}{2 \pi \lambda\left[x-x_{\omega}\right] \sin \left(2 \pi \lambda x_{\omega} / x\right)}\right) d x
$$

where

$$
x_{\omega}=\frac{\left(\omega+\omega_{0} Q_{0}\right) p_{0}}{\omega_{0} \sigma_{p}|\eta \lambda|}
$$

The integral can be used to draw a threshold diagram i.e. a mapping of line $\operatorname{Im} x_{\omega} \rightarrow+0$ on a complex plane. Righthand part of this symmetric curve is plotted in Fig. 3 at several $\lambda$. It is seen that the coasting beam approximation is acceptable at least at $\lambda>10$. Additionally, one can use the formula:

$$
\frac{1}{\sqrt{2 \pi}} \int_{-\infty}^{\infty} \frac{e^{-x^{2} / 2}}{x-x_{\omega}} d x \simeq-\frac{x_{\omega}}{x_{\omega}^{2}-1}+i \sqrt{\frac{\pi}{2}} e^{-x_{\omega}^{2} / 2}
$$

which provides rather good accuracy at $x_{\omega}>2$. Then, for frequent case of dominated space charge impedance, the dispersion equation can be put in a more convenient form:

$$
\begin{gathered}
\frac{r_{0} p_{0} N Z^{(s c)}}{2 \pi \gamma \sigma_{p} Q_{0} Z_{0}\left|\left(k-Q_{0}\right) \eta+\xi\right|}=\frac{x_{\omega}^{2}-1}{x_{\omega}} \\
\frac{\operatorname{Re} Z_{k}^{\perp}}{Z^{(s c)}}=\sqrt{\frac{\pi}{2}} \frac{x_{\omega}^{2}-1}{x_{\omega}} e^{-x_{\omega}^{2} / 2}
\end{gathered}
$$

Left-hand part of Eq. (18) is simply the ratio of r.m.s. frequency spread to the space charge tune shift. Right-hand part should be determined by solution of Eq. (19) typically being about $3-5$. After that, threshold of instability of $k$ th harmonic can be found from Eq. (18). Minimal of the partial thresholds is the ultimate intensity of the beam.

\section{RECYCLER DAMPER}

Now we are applying the results obtained to determine requirements to the Recycler damper. The following parameters are used: $\gamma=9.526, Q_{0}=25.425, \eta=$ $-0.00851, Z^{(r w)}=18 \mathrm{MOhm} / \mathrm{m}$ (stainless steel pipe $9.8 \times$ $4.6 \mathrm{~cm}^{2}$ ). Space charge impedance is taken as large as 500 $\mathrm{MOhm} / \mathrm{m}$ corresponding normalized transverse emittance $1 \pi \mathrm{mm}$-mrad. Longitudinal r.m.s. emittance is $15 \mathrm{eV}$-s corresponding $\sigma_{p}=0.678 \mathrm{MeV} / \mathrm{c}$. Total impedance is calculated by Eq. (12) at $g=2.64$ which is double minimal gain.

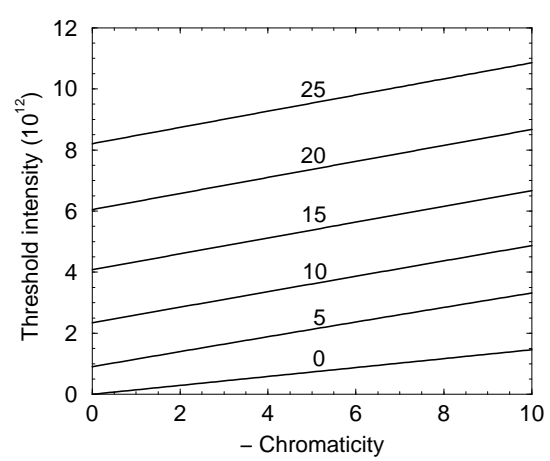

Figure 4: Threshold of instability in the Recycler. Numbers mean the damper bandwidth in $\mathrm{MHz}$ ( 0 - no damper).

Results are presented in Fig. 4 where threshold of instability is plotted against the chromaticity and the damper bandwidth $W=\omega_{0} / 2 \pi \alpha$. Without the damper and at nominal chromaticity $\xi=-2$, the threshold is about $3 \times 10^{11}$ what is confirmed by experiment [2]. At zero chromaticity, the threshold is less by factor $\sim 400$. Above the threshold, increment of the lower unstable mode is:

$$
\operatorname{Im} \omega \simeq 37 \times 10^{-12} N B^{-1 / 3}\left(\mathrm{~s}^{-1}\right)
$$

what concurs with experiment also. A planned intensity $6 \times 10^{12}$ can be reached with $18 \mathrm{MHz}$ damper at nominal chromaticity and with $13 \mathrm{MHz}$ one at chromaticity -10 .

\section{CONCLUSION}

Modification of general theory of bunched beam transverse instability allows to get simpler equations for a rectangular bunch. Their numerical solution shows that, at given longitudinal emittance, the bunch is most inclined to the instability at $B=1$. In this case there is no big difference between barrier-bunched and coasting beam, at least for high harmonics and/or at small slippage factor. The techniques developed are applied to explain transverse beam instability in the Fermilab Recycler Ring, and to develop requirements to its damping system. It is shown that the damper bandwidth about $20 \mathrm{MHz}$ is enough to reach planned beam intensity $6 \times 10^{12}$ ppp at nominal chromaticity.

\section{ACKNOWLEDGMENTS}

Author thanks A. Burov, V. Lebedev, and S. Nagaitsev for fruitful discussion.

\section{REFERENCES}

[1] Gerry Jackson, “The Fermilab Recycler Ring Technical Design Report”, Fermilab-TM-1991 (1996).

[2] Martin Hu, James L. Crisp "Fermilab Recycler Damper Requirements and Initial Design". These Proceedings, MPPP011

[3] F. Sacherer, "Methods for Computing Bunched-Beam Instabilities", CERN-SI-BR-72-5 (1972).

[4] A. Piwinski, "Impedances in Lossy Elliptical Vacuum Chamber”, DESY 94-068 (1994). 